\title{
Diacronie
}

Studi di Storia Contemporanea

$N^{\circ} 14,2$ | 2013

Processo penale, politica, opinione pubblica (secoli XVIII-XX)

\section{Sacco e Vanzetti: caso giudiziario o affaire?}

\section{Flavia Tudini}

\section{(2) OpenEdition \\ Journals}

\section{Edizione digitale}

URL: https://journals.openedition.org/diacronie/160

DOI: $10.4000 /$ diacronie. 160

ISSN: 2038-0925

Editore

Association culturelle Diacronie

Notizia bibliografica digitale

Flavia Tudini, «Sacco e Vanzetti: caso giudiziario o affaire?», Diacronie [Online], № 14, 2 | 2013,

documento 4, online dal 01 août 2013, consultato il 21 septembre 2021. URL: http://

journals.openedition.org/diacronie/160 ; DOI: https://doi.org/10.4000/diacronie.160 


\section{Diacronie}

\section{Sacco e Vanzetti: caso giudiziario o affaire?}

Flavia TUDINI*

In questo articolo si prende in considerazione forse il più eclatante caso giudiziario dell'America d'inizio Novecento: è il caso Sacco e Vanzetti. Il reato di cui sono accusati, le fasi processuali e la loro tragica fine sono noti a tutti, ma si può dire lo stesso delle strategie difensive dei loro avvocati? L'analisi di queste e le modalità in cui si manifesta l'intervento dell'opinione pubblica permettono di definire questo caso giudiziario un affaire con una portata internazionale dirompente, sottovalutata dalle stesse autorità americane. Le vicende giudiziarie verranno mitizzate dall'opinione pubblica che renderà $i$ due anarchici, schiacciati dall'ingiustizia e da un processo politico, simboli della lotta politica antistatale.

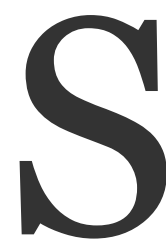

acco e Vanzetti: il loro caso è diventato un affaire di portata molto più ampia del processo che li ha visti protagonisti e vittime nell'America del primo Novecento. Simbolo d'ingiustizia e repressione, la loro vicenda resta una ferita non cicatrizzata nella storia americana.

\section{Odio razziale e red scare}

Tra il 1865 e il 1915, circa 26 milioni d'immigrati giungono in America dall'Europa sud-orientale. Le caratteristiche di quest'ondata migratoria creano subito diffidenza e sospetto negli americani di più antico insediamento. Gli immigrati non conoscono la lingua, sono cattolici, poco istruiti e occupano i livelli più bassi del mercato del lavoro. La povertà e la fame di lavoro dei nuovi arrivati permette, quindi, lo sfruttamento di quest'ondata umana, sottoposta a vessazioni e salari miseri, che sembra non ribellarsi pur di mantenere un impiego. Parallelamente sorgono nelle città i quartieri etnici, dove gli immigrati vivono in ambienti malsani e affollatissimi; in cui però si tramandano le 
tradizioni nazionali, si sviluppa un micro commercio e si svolgono attività culturali. Negli anni Dieci del Novecento, i nuovi immigrati riescono ad inserirsi meglio nella società americana, arrivando a diventare operai specializzati, partecipando alle lotte operaie o politiche, ma talvolta anche avvicinandosi ad attività criminose. Tutto ciò accresce la diffidenza nei loro confronti, fa emergere sentimenti xenofobi e movimenti razzisti che rifiutano qualsiasi ipotesi di accoglienza e melting pot in nome di ideali di purezza della razza anglosassone, minacciata dalle razze inferiori, meno intelligenti e assai prolifiche ${ }^{1}$.

Questa situazione delicata, di difficile integrazione e sospetto razzista, si acutizza in coincidenza della Prima guerra mondiale e del primo dopoguerra. La guerra mette in luce le fratture profonde di natura etnica e sociale presenti nel Paese; moltissimi sono i renitenti all'iscrizione nei registri di leva (Selective Service Act, 1917), nelle industrie belliche si susseguono violenti scioperi e si teme che i nazionalismi degli immigrati riemergano. Il governo federale, per cementare il fronte interno, utilizza un'abile propaganda e impone anche dure leggi repressive, restringendo fortemente il diritto di parola: ogni dissenso è bollato come antipatriottico e antiamericano (Sedition and Espionage Act, 1917); si cerca di uniformare il pensiero ed il comportamento dei cittadini attraverso una profonda opera di americanizzazione e con la persecuzione del movimento operaio. Espressione di quest'ostilità è l'atteggiamento delle élites americane, spaventate dalla Rivoluzione russa. La paura che gli scioperi diventino l'anticamera della rivoluzione anche negli USA si diffonde, acquistando sempre maggiore concretezza nell'immediato dopoguerra, caratterizzato da una congiuntura economica negativa. Da quel momento ha inizio la red scare (la paura dei rossi); il governo federale cercherà di prevenire, con metodi al limite della legalità, il diffondersi dell'ideologia sovversiva e radicale ${ }^{2}$.

La red scare raggiunge l'apice di violenza negli anni 1919-1920, a seguito di diversi attentati dinamitardi anarchici. Tra le alte personalità prese di mira si annovera anche il Ministro della Giustizia Palmer, che a seguito di un fallito attentato presso la sua casa di Washington, dà inizio ad una spirale di persecuzioni, i Palmer raids, rivolti verso tutti coloro che vengono considerati sovversivi; crea anche una General Intelligence Division, una centrale organizzativa contro i radicali affidata a Edgar Hoover. Gli agenti irrompono in uffici, sezioni e centri culturali gestiti da sospetti, arrestano migliaia di persone, molte delle quali saranno poi rimandate nei Paesi di origine; le garanzie costituzionali sembrano essere sospese per far fronte all'emergenza rivoluzionaria.

${ }^{1}$ TESTI, Arnaldo, Il secolo degli Stati Uniti, Bologna, Il Mulino, 2008, p. 116.
2 BERGAMINI, Oliviero, Storia degli Stati Uniti, Bari, Laterza, 2009, p. 156. 
Parallelamente si conducono le indagini sul fallito attentato al Ministro Palmer; la pista degli anarchici sembra essere quella più attendibile, anche a causa di alcune informazioni che aiutano a individuare la tipografia dove sono stati pubblicati i volantini rinvenuti sui luoghi di diversi attentati. A New York il 25 febbraio 1920 sono arrestati Elia e Salsedo, tipografi appartenenti ai circoli anarchici. I due sono trattenuti, senza alcuna assistenza legale, per otto settimane negli uffici del Ministero della Giustizia; per ottenere una confessione, nella più grande segretezza, sono probabilmente sottoposti a pressioni o torture. All'alba del 2 maggio il corpo di Salsedo viene trovato sul selciato della strada, completamente sfigurato, in corrispondenza della finestra della sua cella. È un suicidio o un omicidio? I circoli anarchici considerano questa morte un omicidio di Stato, l'inizio di una repressione ancora più dura e violenta che non avrebbe risparmiato nessuno3. In questa circostanza si trova per la prima volta menzione di Vanzetti, delegato del circolo anarchico di Boston, che è giunto a New York con il compito di procurarsi notizie riguardo all'arresto dei due tipografi, e che dopo la morte di Salsedo torna a casa con il compito di far sparire dalla circolazione qualsiasi tipo di propaganda anarchica finché la situazione non si sia calmata.

\section{Le rapine}

Il 24 dicembre 1919 a Bridgewater (Massachussets) alcuni impiegati del calzaturificio White Shoe Company stanno trasportando le paghe della settimana. Improvvisamente compare una Hudson Overland da cui scendono tre uomini a volto scoperto, che tentano di fermare il veicolo. La sparatoria è inevitabile ma, grazie alla prontezza degli impiegati, la risposta è immediata. La rapina non è andata a buon fine e vi sono diversi testimoni che potrebbero riconoscere gli assalitori.

Alcuni mesi dopo, 15 aprile 1920, a South Braintree (Massachussets) il cassiere del calzaturificio Slater\&Morrill, Parmenter, e la guardia Berardelli stanno portando, a piedi, le paghe della settimana agli operai. Ad un tratto vengono accostati da due uomini, a volto scoperto, che li immobilizzano e subito dopo gli sparano a bruciapelo. Tutto avviene in pochi secondi. Dopo aver preso il denaro, salgono su una Buik, con altre due persone a bordo, partendo immediatamente. L'azione è rapidissima e tra $\mathrm{i}$ molti testimoni non tutti possono affermare di riconoscere gli assalitori. Questa volta la rapina è riuscita, ma ha lasciato dietro di sé due cadaveri.

3 ORTNER, Helmut, Sacco e Vanzetti; una tragedia americana, Frankfurt am Main, Zambon, 1996, p. 89. 
Le indagini sulle rapine sono affidate al capo della polizia di Bridgewater, Stewart, che individua diverse analogie tra le due. Benché le prove non manchino, dai bossoli dei proiettili alle testimonianze oculari, inizialmente non è possibile identificare $\mathrm{i}$ rapinatori. Una possibile soluzione è offerta dall'individuazione di un gruppo di anarchici italiani che vivono nella campagna tra le due città. Per Stewart, accecato dal pregiudizio razziale e dall'odio verso i sovversivi, essi sembrano i colpevoli ideali: i circoli anarchici sono sempre alla ricerca di finanziamenti, secondo alcuni testimoni i rapinatori sono immigrati, e una Buik abbandonata è stata trovata in un boschetto in prossimità della casa di uno di loro. Le circostanze sono più che sospette e lo stesso comportamento degli anarchici sembrerebbe confermarle. Uno di loro, Coacci, ha lasciato il paese in tutta fretta, senza dare spiegazioni. Un altro, Boda, possiede una Hudson Overland che ha recentemente portato dal meccanico. L'unico modo per confermare i sospetti è trovarlo e farlo confessare, ma l'uomo è sparito, consapevole di essere ricercato. Per fermarlo l'occasione ideale è il ritiro dell'auto4.

Il 5 maggio 1920, Boda con alcuni amici, Orciani, Sacco e Vanzetti, si presenta in officina per ritirare l'auto. Si rende subito conto, però, che qualcosa non va: il nervosismo del meccanico, le scuse addotte per non consegnare l'auto lo insospettiscono; si allontana con i compagni in tutta fretta. La polizia, ormai allertata, non tarda a individuare il gruppetto e si mette sulle loro tracce. I primi ad essere arrestati sono Sacco e Vanzetti, fermati sul treno diretto a Brokton con motivazioni molto vaghe: sono «individui sospetti». Giunti al posto di polizia vengono interrogati da Stewart al quale rispondono evasivamente; sono armati ma cercano di nasconderlo, affermano di non conoscere Boda, cercano di occultare alcuni volantini propagandistici. Successivamente vengono interrogati anche dal procuratore Katzman, al quale continuano a dare risposte evasive, contraddittorie e, in alcuni casi, false. Per due giorni sono trattenuti senza assistenza legale, convinti di essere stati arrestati per possesso illegale di armi e motivi politici. Per questo mentono, sanno che potrebbero essere espulsi e non vogliono tradire altri compagni ${ }^{5}$. Solamente qualche giorno dopo è comunicato loro il motivo dell'arresto: aver partecipato alle rapine di Bridgewater e South Braintree. Essi presentano subito i loro alibi per entrambi i fatti. Sacco il 24 dicembre lavorava in calzaturificio ed il 15 aprile era al consolato italiano per richiedere il passaporto per rimpatriare; Vanzetti in entrambe le occasioni vendeva pesce a Plymouth. L'unico alibi accolto dal procuratore è quello di Sacco del 24 dicembre. Sono

${ }_{4}^{4}$ ADDUCCI, Giovanni, Sacco e Vanzetti; una storia infinita, Roma, Associate, 2005., pp. 100102

5 SCHIAVINA, Roberto, Sacco e Vanzetti, cause e fini di un delitto di Stato, Roma, Samonà\&Savelli, 1970, p. 12. 
quindi mostrati a testimoni chiamati ad identificarli, senza la procedura di confronto; vengono perfino costretti a simulare i comportamenti dei banditi. È una procedura fortemente voluta da Katzman, ma illegale. Pur tra mille incertezze e contraddizioni, alcuni testimoni li riconoscono. Sono quindi incriminati formalmente: Vanzetti per la rapina a Bridgewater, entrambi per i fatti di South Braintree.

\section{Un ingiusto processo 6}

Il 20 giugno 1919 Vanzetti è condotto davanti alla corte di Plymouth per rispondere di tentata rapina e tentato omicidio; presiede il giudice Thayer. La scelta del procuratore Katzman di procedere così celermente contro Vanzetti è dovuta alla volontà di ottenere una condanna prima dell'inizio del processo per i fatti di South Braintree, in modo da presentare alla corte uno degli accusati come pregiudicato, nel caso non siano sufficienti le prove addotte. La strategia dell'avvocato della difesa, incentrata sulla conferma dell'alibi dell'imputato da parte dei suoi clienti della città, si rivela poco incisiva. Tutti i testimoni sono italiani di umili origini, che vengono abilmente intimiditi e screditati da Katzman, mentre testimoni dell'accusa, pur caduti più volte in contraddizione, non sono mai messi in discussione dalla corte. Il 16 agosto il giudice condanna Vanzetti alla reclusione nel carcere di Charlestown per un periodo compreso tra i dodici e i quindici anni.

Il 31 maggio 1921 si apre a Dedham il processo contro Sacco e Vanzetti accusati dei fatti di South Braintree7; l'istruttoria è durata un anno, a causa delle dichiarazioni contraddittorie dei testimoni, che faticano a identificare Sacco come uno dei banditi. Nuovamente presiede la corte il giudice Thayer; gli avvocati della difesa sono Moore ed i fratelli McArney, l'accusa è sostenuta dal procuratore Katzman. Le fasi iniziali del processo riguardano solo la rapina, senza entrare nel merito della morte dei due impiegati; si tratta di identificare i banditi. Al proposito si procede all'escussione dei testimoni dell'accusa e della difesa, focalizzando il contraddittorio sulla loro credibilità. L’incertezza e la contraddittorietà di alcuni testi dell'accusa, evidenti prima del processo, sembrano in questa sede scomparire. Le dichiarazioni sono precise, ricche di particolari e prive di dubbi; nel caso delle molte incongruenze il procuratore soprassiede, anzi incita la corte a tenere conto della buona fede dei testi. Sacco e Vanzetti sono riconosciuti, seppur tra qualche incertezza. La difesa cita come testimoni

\footnotetext{
${ }^{6}$ SELLERS, Aldous, BROWN, Arthur, Il caso Sacco e Vanzetti, Roma, Gherardo Casini editore, 1997, pp. 102 et seq.

7 Ibidem.
} 
per Sacco un funzionario del consolato e i conoscenti che vi ha incontrato nelle stesse ore della rapina; per Vanzetti i suoi stessi clienti. Tutti sono però ritenuti poco credibili dal procuratore: sono amici e conoscenti dei due imputati, tutti italiani, e quindi tendenzialmente portati a mentire per proteggerli. In queste prime udienze si evidenziano la tendenziosità delle domande di Katzman e le sue pressioni sulla corte per far riconoscere come valide solo le testimonianze dell'accusa, in alcuni casi fortemente contraddittorie. Nella seconda fase del processo la discussione si sposta sulla morte della guardia. Il procuratore intende dimostrare che la pistola di Sacco è stata usata per ucciderla e che la seconda pistola trovata in possesso degli imputati era stata sottratta alla vittima dopo la morte. Le prove a carico sono fornite da periti balistici del tribunale. Uno di essi sostiene in modo molto ambiguo che il proiettile fatale è stato sparato dall'arma in esame, senza però specificare se si riferisca alla pistola che materialmente è citata come prova o al modello della stessa. La difesa si limita ad opporre a queste vaghe dichiarazioni due suoi periti, che non riescono ad essere sufficientemente convincenti ${ }^{8}$. Infine per dimostrare la colpevolezza dei due imputati Katzman ricorre all'uso probatorio della consapevolezza della colpa; in base alla quale un reo cosciente della sua colpevolezza tiene un comportamento sospetto, ambiguo e contraddittorio ${ }^{9}$. Le prove della condotta colpevole sono legate alle circostanze dell'arresto; essere andati con Boda a ritirare l'auto, aver tenuto un comportamento contraddittorio nel momento dell'arresto, aver mentito e dichiarato il falso nel primo interrogatorio. Per smontare l'impianto accusatorio, la difesa decide di interrogare gli stessi imputati sulla loro fede politica per sottolineare come essi fossero intimoriti soprattutto dalla prospettiva dell'arresto per motivi politici.

Il duplice omicidio di South Braintree scivola quindi in secondo piano; da quel momento si processano le opinioni politiche dei due imputati, causa e spiegazione dell'atto criminale. Katzman riesce ad eccitare i sentimenti patriottici ed i pregiudizi della corte, illustrando le idee sovversive dei due anarchici, la loro renitenza alla leva e le loro critiche al sistema capitalistico americano. Al momento della requisitoria il procuratore leva un appello contro gli stranieri. Anche il riepilogo del giudice è pervaso da nazionalismo e pregiudizio; egli, anziché ricapitolare gli elementi di prova che la giuria avrebbe dovuto prendere in considerazione, entra nel merito con giudizi di valore, insiste sulla consapevolezza della colpa, e non evidenzia le contraddizioni nelle prove dell'accusa. Nonostante gli elementi d'incertezza e i vistosi vizi procedurali, il 14

\footnotetext{
8 ORTNER, Helmut, op. cit., p. 112.

9 FRANKFURTER, Felix, «The case of Sacco and Vanzetti» in The Atlantic Magazine. URL: <http://www.theatlantic.com/magazine/archive/1927/o3/the-case-of-sacco-andvanzetti/306625/> [Consultato il 5 maggio 2013].
} 
luglio 1921 la giuria pronuncia la sentenza di condanna a morte per entrambi gli imputati.

Quello che intercorre tra l'estate del 1921 e l'estate del 1927, è un periodo in cui gli avvocati della difesa, prima Moore, poi Thompson, presentano ben otto mozioni di riapertura del processo e diverse richieste di appello, basate sulla riproposizione dei testi che nel primo processo erano apparsi contraddittori, o sulla scoperta di nuovi fatti ritenuti rilevanti. Fino al 1925 le istanze riguardano alcune dichiarazioni dei principali testi dell'accusa che affermano di aver subito pressioni dal procuratore perché riconoscessero gli imputati che non erano stati identificati dopo l'arresto. Nonostante queste gravi accuse, il giudice respinge tutte le mozioni, a causa di ulteriori ritrattazioni dei testimoni. Tra le dichiarazioni giurate a favore della difesa risaltano quelle di due ex funzionari del Governo Federale, che dimostrano come il processo sia stato influenzato dal Dipartimento di Giustizia in chiave politica. Già da tempo il Dipartimento conosceva Sacco e Vanzetti come sovversivi e cercava elementi per espellerli dal Paese; l'occasione adatta si presenta proprio con le due rapine del 1919-1920. Si svela l'esistenza di contatti tra il Governo, la polizia e il procuratore Katzman, che non smentirà mai questi fatti, anzi accusa gli ex funzionari di violare segreti governativi. Thompson da parte sua interviene con un'orazione vibrante in cui si chiede se sia democrazia considerare sacri i segreti di Stato piuttosto che la vita dei cittadini. Nessun elemento, comunque, induce Thayer a rivedere il processo: neanche le dichiarazioni di Madeiros, un comune criminale condannato per rapina e omicidio, che il 16 novembre 1925 confessa la sua partecipazione alla rapina di South Braintree scagionando completamente Sacco e Vanzetti. Sebbene Madeiros risponda a tutte le domande riguardanti il suo ruolo nei fatti, non fa mai i nomi dei complici. Questa reticenza è la causa del rigetto dell'istanza basata sulla sua confessione; il giudice è convinto di aver trovato un complice di Sacco e Vanzetti, non la verità. Per questo il 9 aprile 1927 il giudice Thayer emette la sentenza, confermando la colpevolezza e la pena di morte per i due imputati. Ciò che resta ai due anarchici per poter ancora sperare è un appello alla Corte Suprema del Massachussets o la richiesta di revisione direttamente al Governatore Fuller. Questi a maggio comincia ad interessarsi al caso, sotto la pressione dell'opinione pubblica, creando una commissione speciale composta da giuristi per una revisione preliminare. Il pronunciamento, dopo mesi di udienze, è negativo: i membri, esponenti dell'elite conservatrice di Boston, non hanno trovato elementi sufficienti a riaprire il caso. Sconfortato e deluso dal sistema giudiziario, Thompson lascia l'incarico, che viene affidato all'avvocato Musmanno. Vane sono le successive richieste di appello 
alla Corte Suprema dello Stato, le istanze di revisione alla Corte Suprema Federale e la richiesta di grazia al Presidente degli Stati Uniti.

Sacco e Vanzetti vengono giustiziati il 23 agosto 1927.

\section{Sacco e Vanzetti: dal caso all'affaire ${ }^{10}$}

Quando si parla del caso Sacco e Vanzetti, si richiamano immediatamente alla mente concetti quali processo politico, ingiustizia e diritti negati. È però necessario far notare che un caso giudiziario con caratteristiche politiche non diventa necessariamente un affaire: è necessario che se ne verifichino le condizioni. Cercare di crearle è storicamente compito degli intellettuali e degli avvocati della difesa, che utilizzano per questo fine gli strumenti del diritto e la propaganda ${ }^{11}$. L'affaire implica che le finalità politiche del processo non siano generalmente evidenti all'opinione pubblica. Il compito degli intellettuali e dei giuristi è quindi quello di svelare i retroscena del processo e di sensibilizzare l'opinione pubblica, per denunciarne le caratteristiche politiche e la violazione dei diritti dell'imputato. L'intento è di rendere nota all'intera società l'uso strumentale del diritto fatto dall'establishment politico e giudiziario, che attraverso la forza della legge vuole liberarsi di elementi socialmente o politicamente scomodi ${ }^{12}$. Solo comprendendo questo concetto è possibile interrogarsi sul processo a carico di Sacco e Vanzetti, e sulle modalità che hanno usato gli avvocati della difesa per rendere un processo per rapina e duplice omicidio una cause celébre.

Come si passa dal caso Sacco e Vanzetti all'affaire? Già durante il processo di Plymouth contro Vanzetti per la tentata rapina di Bridgewater, i comitati anarchici di Boston e Felicani, redattore del giornale «La Notizia», sono convinti della natura politica del processo appena concluso e di quello che si sarebbe aperto a breve per i fatti di South Braintree. A questo proposito Felicani decide di informare diffusamente l'opinione pubblica su quanto sta accadendo, non come gli altri giornali di Boston che alla vicenda dedicano poco più di qualche trafiletto nelle ultime pagine. Egli ritiene che la natura politica di questo processo sia pregna di conseguenze e significato per tutti gli immigrati con opinioni politiche. Comincia quindi a scrivere e pubblicare finte lettere di lettori, provenienti dalle maggiori città d'America, per far credere che ovunque ci siano persone indignate per l'accusa e l'andamento del processo. Dopo la condanna di

10 TEMKIN, Moshik, The Sacco and Vanzetti Affair; America on trial, New Haven, Yale University Press, 2009, p. 15.

${ }^{11}$ CLAVERIE, Elisabeth, «Proces, affaire, cause; Voltaire e l'innovation critique» in Politix. Revue des sciences sociales du politique, 26, 7/1994.

${ }^{12}$ Si veda: ISRAËL, Liora, Le armi del diritto, Milano, Giuffrè, 2012. 
Vanzetti, effettivamente iniziano ad arrivare molte vere dichiarazioni di simpatia da parte dei lettori. Parallelamente nascono i primi comitati finalizzati alla raccolta dei fondi per pagare gli avvocati in vista del processo di Dedham. Felicani ha il difficile compito di coordinare le attività dei diversi sostenitori (i sindacati, i socialisti, gli anarchici, i sostenitori dei diritti civili, con la sola esclusione dei comunisti) accumunati solo dalla lotta per i due anarchici ${ }^{13}$. Questo non è però sufficiente per trasformare il caso giudiziario in affaire; l'interessamento dell'opinione pubblica non basta fintanto che sono coinvolti solo parte dei lavoratori di tendenza radicale e parte degli immigrati italiani. Le élites intellettuali e liberali non sono ancora intervenuti nel movimento a sostegno dei due accusati.

Si resta ancora nella dimensione del caso anche dopo l'affidamento dell'incarico della difesa all'avvocato Moore, un personaggio del quale è necessario introdurre la figura e la strategia difensiva. Egli è un avvocato radicale e lontano dalle regole borghesi della propria corporazione, ha un atteggiamento bohémien e conduce una vita molto chiacchierata, anche a causa delle sue stravaganze nel vestire e dei comportamenti poco ortodossi tenuti durante le udienze. Le sue simpatie politiche lo avevano portato in precedenza ad occuparsi della difesa di diversi sindacalisti dell'Industrial Workers of the World; il caso più conosciuto era stato quello dei sindacalisti italiani Ettor e Giovannitti, accusati di omicidio durante lo sciopero del 1912 a Lawrence, prosciolti poi da ogni accusa. Dopo la vittoria conseguita in questa causa, i radicali e gli immigrati considerano Moore un difensore impegnato nella lotta per i loro diritti; per questo viene scelto da Felicani per patrocinare Sacco e Vanzetti. Accettando l'incarico (agosto 1920), egli si rende conto immediatamente delle finalità politiche del processo. Decide quindi di adottare una strategia difensiva che già in passato aveva funzionato: a class defence aganist a class prosecution ${ }^{14}$, portando il processo dalla corte di Dedham anche davanti a quella che Moore considera la "corte" dell'opinione pubblica; in tal modo egli spera, infatti, di sollevare un'ondata di proteste internazionali. Da un lato s'impegna nella preparazione legale della difesa con l'aiuto dello studio degli avvocati McArney; lui avrebbe formalmente assunto la difesa di Sacco, mentre gli altri sarebbero stati i difensori di Vanzetti. In questo modo avrebbero avuto un controllo più ampio sul processo, potendo utilizzare la facoltà di interrogare due volte i testimoni e di pronunciare due separate arringhe finali. Dall'altro lato, con l'aiuto di Felicani, rafforza e organizza un unico Comitato di Difesa, con lo scopo di

13 ORTNER, Helmut, op. cit., p. 119 et seq.

${ }_{14}$ Si mobilita l'intera classe sociale degli accusati, contrapponendola all'accusa borghese. Cfr. TEMKIN, Moshik, op. cit., p. 25. 
sensibilizzare l'opinione pubblica nazionale ed internazionale attraverso lo svelamento dei retroscena del caso, dimostrando come si stiano processando due uomini non per un reato penale, ma perché italiani, stranieri ed anarchici. L'appoggio è ampio tra gli italiani, i radicali e i sindacalisti, ma esso non è certo sufficiente per creare un'opinione pubblica nazionale. Per fare ciò sarebbe indispensabile ottenere il consenso dal ceto borghese americano, ma questo è assai difficile non solo per il tipo di processo e d'imputati, ma anche a causa del comportamento stravagante e delle idee politiche dell'avvocato Moore che compromettono il successo della sua strategia. A livello internazionale i risultati sono simili: i sostenitori della causa continuano a essere i sovversivi, privi di peso intellettuale e politico, che in diversi Paesi si mobilitano attraverso proteste più o meno pacifiche. Interessante è il ruolo svolto dal giornalista Lyons, inviato in Italia da Moore per creare un movimento d'opinione anche nel paese di origine degli imputati. La missione ha successo specialmente tra gruppi di estrema sinistra e raccoglie l'interessamento dello stesso Mussolini, che vorrebbe utilizzare la vicenda a fini propagandistici sia contro il fragile Stato liberale (perchè non protegge due italiani accusati ingiustamente in terra straniera), sia contro socialisti e comunisti (perché vorrebbe accattivarsi le simpatie degli anarchici per far fronte comune contro i rossi) ${ }^{15}$. Unico caso d'intellettuale coinvolto nella causa fin dal 1921 è quello di Anatole France, che pubblica sul periodico «Nation» un articolo intitolato To the people of America; in questo invoca la liberazione dei due uomini innocenti, arrestati sull'ondata della psicosi successiva alla guerra, richiamando l'affaire Dreyfus come termine di paragone del caso. Data la mancanza di pressioni politiche o diplomatiche, le autorità americane ritengono che l'interessamento internazionale al caso sia legato unicamente ad alcuni specifici gruppi sociali e quindi sia espressione di mera solidarietà di classe. Gli stessi giornali americani non riferiscono le imponenti manifestazioni di protesta in Europa o in America Latina. Questo delinea il fallimento della strategia di Moore: l'opinione pubblica interessata al caso è rimasta circoscritta ad alcuni ambienti sociali, non si è levata l'indignazione borghese e intellettuale necessaria a creare pressione sulla corte. Il caso non è ancora diventato un affaire. Per tutto il restante mandato di Moore l'opinione pubblica colta sembra quindi dimenticarsi dei due anarchici italiani, che nel frattempo vengono condannati.

È possibile parlare di affaire solo a partire dal 23 ottobre 1926, giorno in cui la richiesta di revisione del processo, basata sulla confessione di Madeiros, viene rifiutata dal giudice Thayer. Perché questa decisione del giudice cambia il rapporto tra

${ }_{15}$ CANNISTRARO, Philip V., «Mussolini, Sacco-Vanzetti, and the Anarchists: The Transatlantic Context» in The Journal of Modern History, 68, 1/1996, pp. 31-62. 
l'opinione pubblica e il processo? Cos'è cambiato prima di questa data? Innanzitutto bisogna ricordare che il clima sociale e politico del 1926 è profondamente diverso da quello del 1920: la crescita economica e una nuova legge per l'immigrazione hanno ridotto fortemente la conflittualità sindacale, smorzando in questo modo la paura di un'inevitabile rivoluzione sovversiva anche negli Stati Uniti d'America. Ciò permette ai liberali di osservare in modo più critico la realtà politica, allontanandosi da posizioni estremiste. Questa nuova posizione dei liberali permette quindi a giuristi come Felix Frankfurter un lento interessamento alla causa, che verrà espresso solo dopo il rigetto dell'ultima mozione della difesa. Infine, dopo diverse tensioni tra Moore e Felicani e il fallimento dell'originaria linea difensiva, il Comitato di Difesa rompe il rapporto con quest'avvocato, chiedendo all'avvocato Thompson, di Boston, di sostituirlo. Egli è un avvocato stimato ed influente, sia come docente di giurisprudenza dell'Università di Harvard sia per la sua carriera forense; accetta l'incarico non solo perché ritenga innocenti i due anarchici, ma anche perché avendo osservato la palese violazione della legge a loro danno, ritiene di dover reagire. È, inoltre, un conservatore lontano da qualunque sospetto di vicinanza con idee radicali. Sembra la persona giusta per rivolgersi anche l'opinione pubblica borghese, conservatrice e colta ${ }^{16}$. Fin da subito, infatti, si premura di avvicinare gli ambienti borghesi più conservatori; da questo momento le manifestazioni e le dimostrazioni pubbliche non sono più un affare esclusivo delle organizzazioni operaie.

Tra il 1926 e il 1927 vi è una lenta maturazione della presa di posizione di intellettuali e giuristi riguardo al caso, ma ciò è anche dovuto alla diffusione dell'immagine dei due imputati che si diffonde dal carcere. Soprattutto per quanto riguarda Vanzetti: un lavoratore amante della letteratura e dello studio del pensiero politico del passato, attitudine ancora più stimolata in carcere, dove lo studio è una delle sue consolazioni. Durante il processo e la detenzione, egli si presenta all'opinione pubblica come un abile oratore e un intellettuale: scrive due biografie e un saggio sui sindacati in Italia, studia la letteratura e migliora il suo inglese, riceve moltissime visite e mantiene una fitta corrispondenza. L'immagine che trasmette di sé permette agli intellettuali di avvicinarsi a lui come quasi ad un pari, appassionandosi alla sua sorte. Grazie a quest'immagine, l'opinione pubblica colta, bianca e liberale comincia a interessarsi ai procedimenti giudiziari che coinvolgono i due anarchici. Grazie a Thompson, che trasmette ai suoi colleghi il disagio di trovarsi di fronte al volto sgradevole, assurdo e ingiusto della giustizia americana, anche i giuristi di Harvard cominciano a nutrire interesse per il caso. Tra questi, il primo che prende

${ }^{16}$ ORTNER, Helmut, op. cit., p.197. 
pubblicamente posizione è Frankfurter, che nel marzo 1927 pubblica un articolo intitolato The case of Sacco e Vanzetti sul conservatore "Atlantic Monthly»; in esso sostiene che il processo è stato scorretto e ingiusto, rileva la parzialità del giudice e la collega al clima d'isteria post bellica ${ }^{17}$. È il primo studio del caso da parte un giurista di una certa levatura, per questo e per l'evidente accento politico, l'articolo ha un impatto fortissimo sul pubblico; è inoltre diffuso anche dal Comitato di Difesa e dagli intellettuali anarchici ${ }^{18}$. Da quel momento diversi altri giuristi e professori di Harvard analizzano il caso e prendono posizione, giungendo finanche ad appellarsi al Governatore dello Stato per la riapertura del processo o per la concessione della grazia; anche gli studenti di legge organizzano movimenti di protesta. La controversia assume per la prima volta un altro aspetto, non è più una lotta di classe ma una questione di diritto; si schierano quindi a favore della campagna gran parte delle élites intellettuali, sociali e legali di Boston e degli USA. L'articolo di Frankfurter è diventato potenzialmente più pericoloso delle precedenti dimostrazioni e proteste radicali. Da quel momento il Comitato di Difesa si riorganizza, aprendosi all'ala liberale e progressista del movimento. Nel 1927 i sostenitori sono divisi in due gruppi: i militanti sovversivi, che propugnano azioni dimostrative, politicizzate ed extralegali, e i giuristi e gli intellettuali che continuano a credere nel sistema giudiziario americano. All'interno di entrambi i gruppi vi è un'ulteriore divisione tra coloro che vogliono salvare le vite dei due anarchici a tutti i costi e coloro che invece rimarcano la necessità della giustizia continuando ad oltranza la mobilitazione sociale e politica. All'interno delle proteste degli intellettuali si può annoverare lo scritto di John Dos Passos, Facing the chair; story of the americaniztion of two foreign workman, un memorandum di difesa che attacca soprattutto le procedure processuali, il ruolo del Dipartimento di Giustizia e l'impedimento dei diritti della difesa ${ }^{19}$. Altri intellettuali daranno il proprio contributo alla mobilitazione e, dopo l'esecuzione, alla memoria dei due anarchici sia con testi propagandistici, sia con opere letterarie. Esempi possono essere il testo teatrale con intento documentario di Erich Mühsam Ragion di Stato: una testimonianza per Sacco

\footnotetext{
${ }_{17}$ FRANKFURTER, Felix, «The case of Sacco and Vanzetti» in The Atlantic Magazine, 1 marzo 1927, URL: <http://www.theatlantic.com/magazine/archive/1927/03/the-case-of-sacco-andvanzetti/306625/> [Consultato il 5 maggio 2013].

${ }_{18}$ Tra questi si ricorda: SCHIAVINA, Roberto, Sacco e Vanzetti, cause e fini di un delitto di Stato, Roma, Samonà\&Savelli, 1970 [I edizione: Sacco e Vanzetti, cause e fini di un delitto di Stato, Paris, J. Bucco, 1927].

19 DOS PASSOS, John, Davanti alla sedia elettrica; come Sacco e Vanzetti furono americanizzati, Santa Maria Capua Vetere, Spartaco, 2005 [Ed. originale: Facing the chair; story of the americaniztion of two foreign workman, Boston, Sacco-Vanzetti Defense Committee, 1927].
} 
e Vanzetti ${ }^{20}$, oppure il romanzo di Upton Sinclair Boston ${ }^{21}$ (1927), ispirato alla vicenda. Queste prese di posizione liberali sono però caratterizzate da alcuni limiti, che entrano in contraddizione con le proteste dei radicali. Infatti continuano a credere nel sistema e si astengono da qualunque tipo di manifestazione, in quanto a differenza dei radicali, non vogliono considerare il caso come denuncia dei problemi sociali e giuridici dell'America capitalista.

Perfino in Europa e nell'America Latina, tra il 1926 e il 1927, il caso diventa affaire grazie all'interessamento di gruppi non appartenenti alla classe lavoratrice, alle comunità italiane e alla sinistra radicale. Il nuovo orientamento dell'opinione pubblica anche in questo caso è influenzato dalla pubblicazione e dalla diffusione dello studio di Frankfurter. Diventa evidente in tutto il mondo che negli Stati Uniti d'America si sta per consumare una tragedia, che l'ingiustizia a breve porterà due innocenti alla morte. Importanti figure pubbliche e intellettuali mandano petizioni e appelli al governatore Fuller e al Presidente Coolidge. Tra le personalità che intervengono si ricordano Albert Einstein, Paul Löbe (Presidente del Reichstag), Maximilien Harden, Thomas Mann e anche Alfred Dreyfus; gli stessi ambasciatori americani nel mondo cercano di fare pressione sul proprio governo senza risultati. Più passano i giorni più la situazione diventa drammatica e si susseguono manifestazioni di protesta, spesso violente, a cui i governi non riescono, o non vogliono, far fronte. È possibile affermare che nel 1927 scoppia per la prima volta in Europa un fortissimo sentimento anti-americano, che sarà eguagliato solo dalle proteste europee contro la guerra in Vietnam. Per brevità non si prenderanno in considerazione tutte le manifestazioni a livello internazionale, ma è interessante far notare come le classi lavoratrici, il movimento radicale e gli stessi governi abbiano interpretato queste manifestazioni in modo strumentale e nazionalista, primo tra tutti il regime fascista in Italia.

L'ultimo elemento che caratterizza la transizione dal caso all'affaire è il coinvolgimento dei leader politici; solo attraverso il loro interessamento, un caso locale può essere elevato ad interesse diplomatico. Il primo e più duraturo intervento politico è quello di Mussolini, prima a livello personale e in seguito come capo di Stato, che fin dal 1921 interviene direttamente nella questione. Il suo atteggiamento è però contraddittorio; da un lato vuole sostenere davanti alla comunità internazionale gli interessi nazionali dell'Italia, dall'altro la repressione interna dei movimenti di sinistra diventa ogni anno più dura. Per questo motivo tutte le manifestazioni a favore dei due

${ }^{20}$ MÜHSAM, Erich, Ragion di stato: una testimonianza per Sacco e Vanzetti, Roma, Salerno editore, 2007 [Ed. originale: Staaträson ein Denkmal für Sacco und Vanzetti, Berlin, Gilde freiheitlicher Bücherfreunde, 1929].

${ }^{21}$ SINCLAIR, Upton, Boston, 2 voll., New York, Albert \& Charles Boni, 1928. 
anarchici sono gestite dall'alto, e quelle spontanee vengono sciolte con la forza; i giornali non possono esprimersi liberamente sulla questione, ma devono riportare le veline composte dal governo. Uno dei motivi che stanno alla base del controllo dell'opinione pubblica su quest'argomento è fornito dalle buone relazioni diplomatiche che l'Italia vuole mantenere con gli Stati Uniti. Gli appelli inoltrati da Mussolini non hanno carattere pubblico, ma è una corrispondenza estremamente riservata rivolta alle alte cariche governative statunitensi. Interessante è l'interpretazione data alle possibili conseguenze dell'esecuzione: Mussolini è convinto che la grazia esortata possa essere concessa solo come uno strumento politico per tacitare ulteriori proteste sovversive in tutto il mondo. L'interesse nazionale deve quindi comportare il controllo dei movimenti di sinistra ${ }^{22}$. Numerose altre figure politiche internazionali intervengono nella vicenda: Stalin, che intende darne un'interpretazione comunista, il futuro primo ministro inglese MacDonald, il futuro primo ministro francese Herriot e altri.

\section{Due affaires a confronto}

Dopo aver mostrato come sia nato l'affaire Sacco e Vanzetti, è ora interessante fare un breve confronto tra questo e l'affaire Dreyfus forse il più celebre caso politico dell'età contemporanea, cui il processo dei due anarchici è stato spesso accostato. In entrambi i casi, gli imputati di gravi crimini proclamano la loro innocenza contestando la sentenza e vengono supportati da un vasto pubblico. Ed entrambi sono esempi di grave ingiustizia perpetrata dallo Stato a danno di singoli individui. Infine in entrambi si nota come siano diffusi nella società sentimenti di pregiudizio razziale, odio politico e timore di una cospirazione a danno dello Stato. Se vi sono importanti analogie che aiutano a definire entrambi i casi come affaires, vi sono anche profonde differenze che non possono essere ignorate. Per prima la condizione intrinseca dei protagonisti: Dreyfus è un uomo non politico accusato di un crimine politico, mentre Sacco e Vanzetti sono due uomini politicizzati accusati di un crimine comune. L'origine differente dei due casi comporta differenti prese di posizione dell'opinione pubblica. Dreyfus è parte dell'elite sociale francese, che facilmente s'indigna per lo scandalo; Sacco e Vanzetti sono degli immigrati, gente comune accusata di furto e omicidio, fatto che non favorisce l'insorgere di moti d'indignazione e protesta in loro difesa da parte degli intellettuali. È significativo che la prima mobilitazione sia nata negli ambienti radicali e tra i lavoratori. Infine, per quanto riguarda la pena inflitta, Dreyfus è

${ }^{22}$ CANNISTRARO, Philip V, «Mussolini, Sacco-Vanzetti, and the Anarchists: The Transatlantic Context» in The Journal of Modern History, 68, 1/1996, pp. 31-62. 
condannato ai lavori forzati a vita, i due anarchici sono condannati a morte. Legata a ciò vi è la possibilità di riabilitazione. Dreyfus ottiene il riconoscimento della sua innocenza e il riscatto sociale; Sacco e Vanzetti saranno giustiziati e solo in anni recenti verrà ammessa l'iniquità del processo senza che vi sia, però, menzione alla loro innocenza ${ }^{23}$.

Per concludere è possibile individuare le linee di continuità tra i due processi politici, ma è anche necessario prendere in considerazione la differente l'influenza che hanno avuto nella società. La Francia post affaire Dreyfus è investita da un rinnovamento politico e sociale, improntato dal rafforzamento dei diritti individuali. Gli Stati Uniti d'America dopo la condanna dei due anarchici non s'interrogano sui limiti della propria democrazia e lasciano irrisolto il problema posto dalla loro esecuzione, che resta ancora oggi uno spazio oscuro nella loro storia.

${ }^{23} \mathrm{Nel} 1977$ il governatore del Massachussets riapre il caso e ammette pubblicamente l'iniquità del processo. Si riabilita la memoria dei due anarchici, senza alcuna menzione alla loro innocenza, che a tutt'oggi non può essere provata oltre ogni ragionevole dubbio. In ADDUCCI, Giovanni, op.cit., p. 270. 


\section{* L'autore}

Flavia Tudini ha conseguito la laurea triennale in Scienze Storiche presso l'Università Roma Tre nel 2012, con una tesi basata sullo studio di una fonte diaristica inedita del Novecento. Frequenta il corso di laurea magistrale in Storia contemporanea presso l'Università di Bologna. URL: < http://www.studistorici.com/progett/autori/\#Tudini >

\section{Per citare questo articolo:}

TUDINI, Flavia, «Sacco e Vanzetti: caso giudiziario o affaire?», Diacronie. Studi di Storia Contemporanea : Processo penale, politica, opinione pubblica (secoli XVIII-XX), 29/08/2013,

URL:< http://www.studistorici.com/2013/08/29/tudini_numero_14/ >

Diacronie Studi di Storia Contemporanea $\beta$ www.diacronie.it

Risorsa digitale indipendente a carattere storiografico. Uscita trimestrale. redazione.diacronie@hotmail.it

Comitato di redazione: Marco Abram - Jacopo Bassi - Luca Bufarale - Alessandro Cattunar - Elisa Grandi - Deborah Paci - Fausto Pietrancosta - Matteo Tomasoni - Luca Zuccolo

Diritti: gli articoli di Diacronie. Studi di Storia Contemporanea sono pubblicati sotto licenza Creative Commons 2.5 .

Possono essere riprodotti a patto di non modificarne i contenuti e di non usarli per fini commerciali. La citazione di estratti è comunque sempre autorizzata, nei limiti previsti dalla legge. 